\title{
Cambios en los hábitos de salud oral en pacientes infectados por el virus de la inmunodeficiencia humana
}

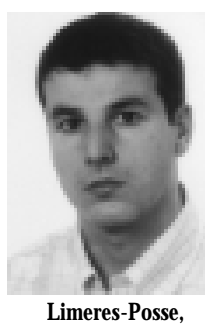
Jacobo

\section{Changes in the oral health habits in patients infected with the human immunodeficiency virus}

Limeres- Posse, J acobo* Tomás-Carmona, Inmaculada* Vázquez-García, Emma* Diz- Dios, Pedro**

*Tutor clínico. Unidad de Pacientes Especiales.

**Profesor titular. Unidad de Pacientes Especiales.

Facultad de Medicina y Odontología. Universidad de Santiago de Compostela

\begin{abstract}
Resumen: Objetivo: Analizar los cambios en la frecuencia de cepillado y demanda de atención odontológica en un colectivo de pacientes VIH-positivos sometidos a seguimiento durante un largo período de tiempo. Pacientes y método: El grupo de estudio lo conformaron 200 individuos VIH-positivos. En 1992 se les realizó una exploración odontológica y una encuesta sobre frecuencia de cepillado dental y visitas al dentista, antes de la seroconversión y en ese año. En 2002, se reexaminó y reencuestó a 56 pacientes, para analizar la actitud de este colectivo hacia los cuidados odontológicos y compararla con la de la población general española. Resultados: La higiene oral mejoró significativamente a lo largo del período de seguimiento (el $45 \%$ del colectivo no se cepillaba diariamente antes de la seroconversión; frente al $25 \%$ en 1992 y al $13 \%$ en 2002). El número de enfermos que visitaba regularmente al dentista aumentó un 15\%entre 1992 y 2002. Conclusión: Los hábitos de higiene y el estado de salud oral de los pacientes VIH-positivos de largo tiempo de evolución son similares a los de la población general.
\end{abstract}

Palabras clave: VIH, SIDA, Higiene oral, Salud oral, Tratamiento odontológico, Odontología.

Abstract: Objective: The aim of this study was to analyse long-term changes in frequency of tooth brushing and dental care request in HIV-infected patients subjected to a longterm follow-up. Patients and method: A group of $200 \mathrm{HIV}$-infected patients underwent oral examination in 1992. They filled out a questionnaire about frequency of tooth brushing and visits to the general dental practitioner before seroconversion and during that year. In 2002, 56 patients were re-examined and re-enquired, allowing us to analyse the attitudes of this cohort towards dental care, and compare them to the Spanish general population. Results: Oral hygiene significantly improved during the follow-up period (45\% of patients brushed their teeth less than once a day before seroconversion; $25 \%$ did so in 1992 and $13 \%$ in 2002). The frequency of regular visits to the dental clinic increased $15 \%$ between 1992 and 2002. Conclusion: Dental hygiene habits and oral health in long-term HIV-positive patients are similar to the general population.

Key words: HIV, AIDS, Dental care, Oral health, Dental treatment, Dentistry.

\begin{tabular}{ccc}
\hline Fecha recepción & Fecha última revisión & Fecha aceptación \\
$12-05-2003$ & $30-10-2003$ & $19-12-2003$ \\
\hline
\end{tabular}

BIBபD [1138-123X (2004)9:2; marzo-abril 125-256]

Lmeres-Posse J , Tomás-Carmona I, Vázquez-García E, Diz-Dios P. Cambios en los hábitos de salud oral en pacientes infectados por el virus de la inmunodeficiencia humana. RCOE 2004;9(2):141-145. 


\section{Introducción}

La aplicación de la terapia antirretroviral de alta eficacia (TAE) en los pacientes infectados por el virus de la inmunodeficiencia humana $(\mathrm{VIH})$, ha aumentado considerablemente su esperanza de vida, considerándose en la actualidad enfermos de carácter crónico ${ }^{1-3}$.

El conocimiento por parte de los pacientes VIH-positivos de su condición de infectados desencadena una mayor preocupación por todo lo concerniente a su salud. Esta actitud afecta secundariamente a los cuidados odontológicos, aumentando la frecuencia de cepillado y la demanda de atención profesional ${ }^{4,5 * *, 6}$.

Todos estos factores podrían presumiblemente condicionar una mejoría de la salud bucodental y de los hábitos de higiene oral en los pacientes VIH-positivos.

El objetivo del presente estudio fue analizar los cambios en los hábitos de salud oral (higiene oral y demanda de atención odontológica) en un grupo de pacientes VIH-positivos sometidos a seguimiento durante un largo período de tiempo.

\section{Pacientes y método}

El grupo de estudio lo conformaron inicialmente 200 pacientes $\mathrm{VIH}$ positivos (143 varones y 57 mujeres; edad media $=36,8 \pm 7$ años) seleccionados aleatoriamente entre los enfermos de la Unidad de Seguimiento de VIH del Hospital Xeral-Cíes (Vigo). En 1992 todos los pacientes cumplimentaron un cuestionario sobre con- sumo de tabaco y drogas, hábitos de higiene oral y frecuencia de visitas al dentista, diferenciando cuando eran VIH-negativos y en el momento de la entrevista. A continuación, se les realizó una exploración intraoral registrando el índice CAO. En 2002 se convocó a este mismo grupo de pacientes para una nueva evaluación: 106 habían fallecido y 38 se habían trasladado o no pudieron ser localizados. Los 56 pacientes restantes, 25 mujeres $(44,6 \%)$ y 31 varones $(55,4 \%$, fueron nuevamente encuestados y examinados (edad media = 44,3 \pm 5 años). Los resultados de los cuestionarios y de las exploraciones odontológicas en 1992 y 2002 fueron recogidos en una base de datos y analizados estadísticamente, permitiéndonos comparar la actitud del mismo colectivo hacia los cuidados odontológicos en términos de: visitas al dentista (nunca/sólo por dolor/al menos una vez al año) y frecuencia de cepillado dental (nunca/a veces/una vez al día/más de una vez al día), en los años 1992 y 2002. Para determinar la evolución de ambas variables, se realizó un análisis de comparación de simetrías (test de McNe$\left.\operatorname{mar}^{7}\right)$. Los datos sobre higiene oral y demanda de atención odontológica obtenidos en el colectivo de estudio en el año 2002 fueron asimismo comparados con los referidos para la población general española del mismo rango de edad ${ }^{8}$.

\section{Resultados}

La conducta de riesgo predominante en el colectivo de estudio fue el consumo de drogas por vía parenteral (CDVP) (66\% de los pacientes), seguida de los contactos heterosexuales (30\%) y de los homosexuales (4\%). En 1992, diez pacientes presentaban una adicción activa a algún tipo de droga (cinco de ellos eran CDVP). En 2002 , siete pacientes (12,5\%) participaban en programas de desintoxicación y recibían metadona, pero ninguno era consumidor activo de drogas recreacionales. En 1992, el 84\% de los pacientes tenía menos de tres años de evolución desde la seroconversión al VIH y en 2002 el 70\%superaba los diez años de evolución. En 1992, el 54\% de los pacientes no recibían medicación antirretroviral y al $46 \%$ se les administraba un único inhibidor de la transcriptasa inversa del VIH. En 2002, el 53\% de los pacientes recibían inhibidores de las proteasas del VIH 1 combinados con inhibidores de la transcriptasa inversa, $28 \%$ estaban tratados exclusivamente con inhibidores de la transcriptasa inversa y el 19\% no recibía ningún fármaco antirretroviral.

El hábito de cepillado dental mejoró tras el diagnóstico de la infección por VIH y continuó con esta evolución favorable entre los años 1992 y 2002. Antes de la seroconversión, el 44,6\% del colectivo de estudio se cepillaba menos de una vez al día, reduciéndose este porcentaje al 25\%en 1992 y al 13,2\%en 2002. Esta tendencia resultó estadísticamente significativa en ambos períodos ( $p<0,001$ y $p<0,005$ respectivamente) (tabla 1 ).

La frecuencia de visitas al dentista aumentó de forma considerable tras conocer los pacientes su condición de infectados por el VIH; esta actitud continuó mejorando entre los años 1992 y 2002 . El porcentaje de pacientes que visitaban al dentista al menos 


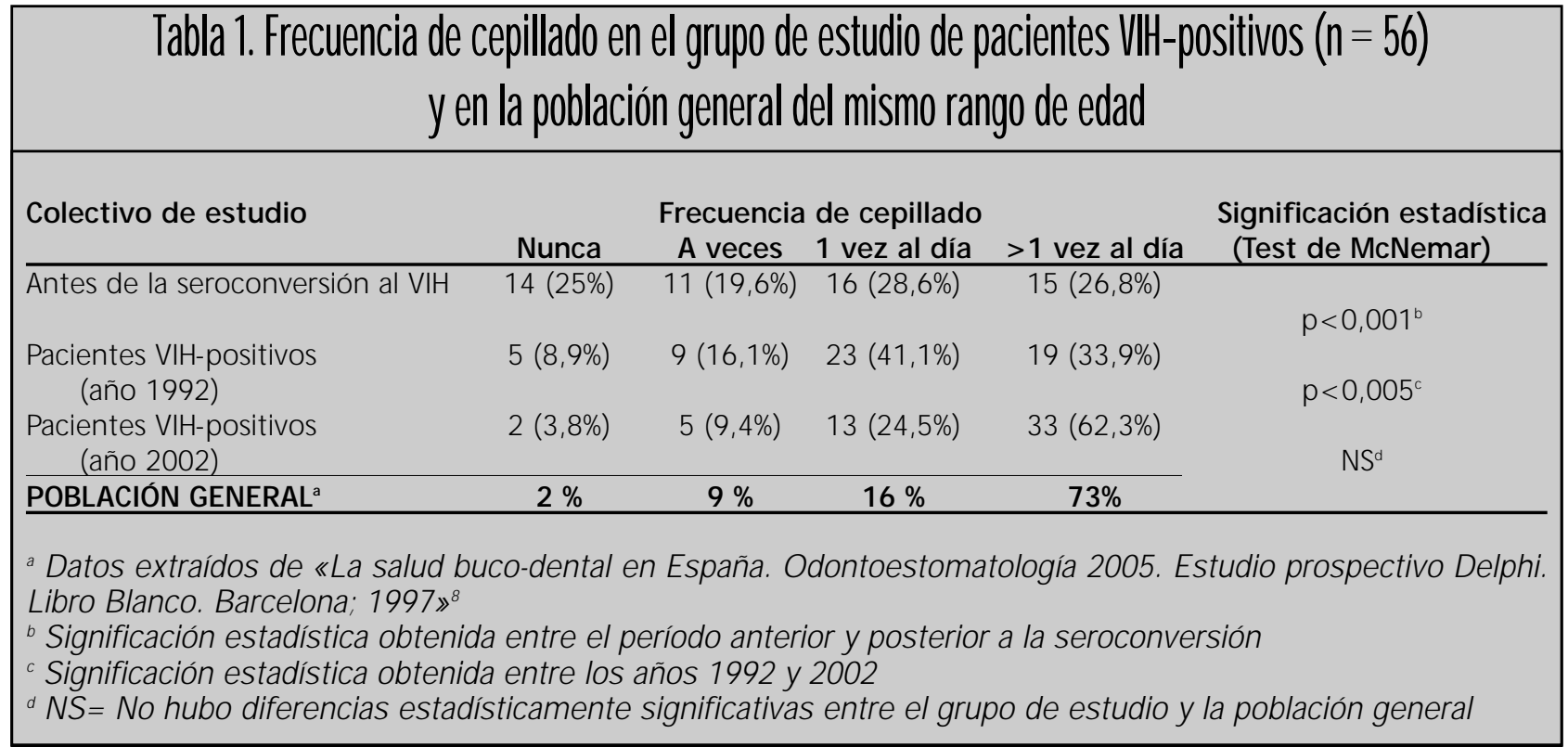

\begin{tabular}{|l|l}
\hline \multicolumn{4}{|c|}{ Tabla 2. Frecuencia de visitas al dentista en el grupo de estudio de pacientes VH-positivos $(n=56)$} \\
y en la población general del mismo rango de edad
\end{tabular}

una vez al año fue del $12,5 \%$ tras la seroconversión, del 21,4\%en 1992 y del $35,7 \%$ en 2002 ( $p<0,005$ y $p<0,01$ respectivamente) (tabla 2 ).

La prevalencia de caries activa descendió del $76,8 \%$ al $53,6 \%$ durante los diez años de seguimiento. El porcen- taje de pacientes con más de seis obturaciones aumentó del 3,6\%al 21,4\% durante el período de seguimiento (tabla 3). Sólo el 7\% de los pacientes tenían algún tipo de rehabilitación protésica en 1992, frente al 35,7\% en 2002.

\section{Discusión}

A diferencia de otros autores 9 , el grupo de estudio se seleccionó entre los pacientes de una consulta médica y no de una clínica dental, ya que en- 
tre éstos últimos se podría presuponer una actitud más positiva hacia los cuidados odontológicos.

La atención odontológica de pacientes infectados por VIH se ha relacionado con la predisposición de los profesionales de la Odontología al tratamiento de estos enfermos, ya que durante los primeros años de la epidemia, se detectó cierta reticencia entre los dentistas hacia el tratamiento de individuos VIH-positivos ${ }^{10}$. Posteriormente se ha demostrado que la actitud del dentista ${ }^{11,12^{* *}}$, el temor de los pacientes a ser rechazados ${ }^{12^{* *}}$ y la falta de información ${ }^{13}$, pueden condicionar una escasa utilización de los servicios de Odontología entre este colectivo. Los dentistas del área geográfica en la que se realizó el presente estudio mostraron una adecuada predisposición hacia el tratamiento de estos enfermos ${ }^{14}$, por lo que consideramos que este factor no ha influido en los resultados obtenidos. También se ha sugerido que algunos condicionantes socioeconómicos (seguro dental, nivel de estudios, etc.) y el modelo sanitario del país, determinan el acceso a los cuidados odontológicos de los pacientes VIH-positivos ${ }^{15-17 * *-20}$. En nuestro estudio ninguno de estos factores se modificó de forma significativa entre 1992 y 2002.

El grupo de estudio estuvo compuesto principalmente por individuos ex-CDVP, como reflejo de las características epidemiológicas de la infección por VH en España en 1992. Este dato podría justificar la elevada prevalencia de caries detectada en la exploración basal. Angelino y cols ${ }^{21}$, en pacientes adictos a heroína encontraron sólo un $6,5 \%$ de pacientes sin caries y Szyma-

\begin{tabular}{|c|c|c|c|c|}
\hline & Ninguna & $<3$ & 3-6 & $>6$ \\
\hline \multicolumn{5}{|l|}{ Caries } \\
\hline año 1992 & $13(23,2 \%)$ & $17(30,4 \%)$ & $16(28,6 \%)$ & $10(17,8 \%)$ \\
\hline año 2002 & $26(46,4 \%)$ & $17(30,4 \%)$ & $7(12,5 \%)$ & $6(10,7 \%)$ \\
\hline \multicolumn{5}{|l|}{ Ausencias dentarias } \\
\hline año 1992 & $10(17,9 \%)$ & $18(32,1 \%)$ & $11(19,6 \%)$ & $17(30,4 \%)$ \\
\hline año 2002 & $8(14,3 \%)$ & $9(16,1 \%)$ & $17(30,4 \%)$ & $22(39,2 \%)$ \\
\hline \multicolumn{5}{|l|}{ Obturaciones } \\
\hline año 1992 & $29(51,8 \%)$ & $13(23,2 \%)$ & $12(21,4 \%)$ & $2(3,6 \%)$ \\
\hline año 2002 & $22(39,3 \%)$ & $15(26,8 \%)$ & $7(12,5 \%)$ & $12(21,4 \%)$ \\
\hline
\end{tabular}

niak y $\operatorname{cols}^{22}$, obtuvieron el doble de caries activas y ausencias dentarias en consumidores de drogas frente a individuos sin este hábito. Algunos autores $^{21-24}$ han sugerido que, en este tipo de pacientes, el grado de patología dental está directamente relacionado con la mala higiene oral y los años de consumo activo de drogas.

La mejora en los hábitos de higiene oral de los enfermos tras la seroconversión al $\mathrm{VIH}$ ya ha sido observada por otros autores ${ }^{4,6}$. En nuestro colectivo de estudio esta tendencia favorable se mantuvo a lo largo del período de seguimiento hasta alcanzar en el año 2002 niveles similares a los referidos en la población general española del mismo rango de edad ${ }^{8}$.

Coincidimos con otros autores $s^{4,5^{* *}, 6}$ al constatar un aumento en el número de visitas al dentista tras la seroconversión. En 2002 la frecuencia de consultas fue similar a la observada en la población general española ${ }^{8}$, y superior a la observada en otros países europeos ${ }^{25}$.

Ter Horst y cols $^{26}$, en un estudio realizado entre 500 dentistas señalaron que los planes de tratamiento eran menos elaborados para pacientes usuarios de drogas que para no consumidores, con una mayor propuesta de obturaciones simples y prótesis removibles frente a prótesis fijas. Este hecho podría justificar el notable número de obturaciones y prótesis de nuestro colectivo de estudio en 2002.

Coincidimos con otros autores ${ }^{27}$ en que las diferencias interindividuales encontradas a lo largo del período de seguimiento, probablemente están relacionadas con factores educacionales y socioeconómicos, reflejando lo que ocurre en la población general. En este sentido, Gallagher y cols ${ }^{28}$ propusieron la realización de campañas de promoción de la salud oral dirigidas a pacientes VIH-positivos. Los resultados de este estudio reflejan que los hábitos de cepillado dental y la demanda de cuidados odontológicos en infectados por el $\mathrm{VIH}$ de largo tiempo de evolución son similares a los de la población general, poniendo en tela de juicio la necesidad de diseñar programas específicos de promoción de la salud oral para este colectivo. 


\section{Bibliografía recomendada}

Para profundizar en la lectura de este tema, el/los autor/es considera/an interesantes los artículos que aparecen señalados del siguiente modo: *de interés $* *$ de especial interés.

1. Bossi P, Martinez V, Strady C, Bricaire F. Current antiretroviral therapy. Rev Med Interne 2002;22:42-52.

2. Rogers PA, Sinka KJ, Molesworth AM, Evans $\mathrm{BG}$, Allardice GM. Survival after diagnosis of AIDS among adults resident in the United Kingdom in the era of multiple therapies. Commun Dis Public Health 2000;3:188-94.

3. The CASCADE Collaboration. Concerted Action on SeroConversion to AIDS and Death in Europe. Survival after introduction of HAART in people with known duration of HIV-1 infection. Lancet 2000;355:1158-9.

4. Vázquez E, Diz P, Feijoo J, Álvarez J, Castro M, Ocampo A. Actitudes de los pacientes VIHpositivos hacia los cuidados odontológicos. Rev Eur Odontoestomatol 1997;1:47-50.

5**. McCarthy GM, Haiji FS, Mackie ID. Attitudes and behaviour of $\mathrm{HIV}$-infected patients concerning dental care. J Can Dent Assoc 1996;62:63-9. En este estudio se analiza la actitud hacia los cuidados odontológicos de los pacientes infectados por el VIH, y se reflejan las dificultades para el acceso al tratamiento.

6. Terry SD, Jones JE, Brown RH. Dental-care experiences of people living with HIV/AIDS in Aotearoa, New Zeland. New Zealand Dent J 1994;62:63-9.

7. Agresti A. Categorical Data Analysis. 1st ed. New York: Wiley \& sons, 1990:353

8. Estudio prospectivo Delphi. Libro Blanco. La salud buco-dental en España. "Odontoestomatología 2005". 1st ed, Barcelona: Lacer, 1997:29-34.

9. Marcenes W, Pankhurst CL, Lewis DA. Oral health behaviour and the prevalence of oral manifestations of HIV infection in a group of HIV positive adults. Int Dent J 1998;48:557-62.

10. Searle ES. Knowledge, attitudes and behaviour of health professionals in relation to AIDS. Lancet 1987;3:26-8.
11. Robinson PG, Croucher R. Access to dental care-experiences of men with HIV infection in the United Kingdom. Community Dent Oral Epidemiol 1993;21:306-8.

$12 * *$. Robinson P, Zakrzewska JM, Maini M, Williamson D, Croucher R. Dental visiting behaviour and experiences of men with $\mathrm{HIV}$. $\mathrm{Br}$ Dent J 1994; 176:175-9.

Estudio realizado en varones VIH-positivos donde se analizan los factores que condicionan el acceso y la demanda de atención odontológica.

13. Shibosky CH, Palacio H, Neuhaus JM, Greenblatt RM. Dental care access and use among HIV-infected woman. Am J Public Health 1999;89:834-9.

14. Vázquez García E, Diz Dios P, Batalla Vázquez P, Castro Ferreiro M, Álvarez Álvarez J, Fernández Feijoo J. Actitudes de los profesionales odontólogos ante el paciente infectado por el VIH. Rev Eur Odontoestomatol 1996;8:277-82.

15. Marcus M, Freed JR, Coulter ID y cols. Perceived unmet need for oral treatment among a national population of HIV-positive medical patients: social and clinical correlates. Am J Public Health 2000;90:1059-63.

16. Green VA, Chu SY, Diaz T, Schable B. Oral health problems and use of dental services among HIV-infected adults. Supplement to HIV/AIDS Surveillance Project Group. J Am Dent Assoc 1997;128:1417-20.

$17 * *$. Coulter ID, Marcus M, Freed JR y cols. Use of dental care by HIV infected Medical Patients. J Dent Res 2000;79:1356-61.

Aunque no totalmente extrapolable a nuestro entorno socioeconómico, este estudio hace un pormenorizado análisis de las variables que condicionan la atención odontológica de pacientes VIH-positivos.

18. Fleishman JA, Schneider DA, Garcia I, Hardwick K. Dental service use among adults with
Human Immunodeficency Virus Infection. Med Care 1997;35:77-85.

19. Maskarenhas AK, Smith SR. Factor associated with utillization of care for oral lesions in HIV disease. Oral Surg Oral Med Oral Pathol 1999;87:708-13.

20. Scheutz F. Dental care of HIV-infected patients: attitudes and behaviour among Danish dentist. Community Dent Oral Epidemiol 1989;17:117-9.

21. Angellino IF, Grasso GM, Sagliocco G, Villari P, D'Errico MM. Dental health in a group of dug addicts in Italy. Community Dent Oral Epidemiol 1991;19:36-7.

22. Szymaniak E, Waszkiel D, Dymokowska W. The condition of teeth and the need for teeth treatment in drug addicts. Czas Stomatol 1990;43:134-9.

23. Silvestre FJ, Bagán JV, Del Olmo JA, Jimeno V. Oral status of drug addicted patients. Study of 66 cases. Actual Odontostomatol (Paris) 1990;44:299-306.

24. Molendijk B, Ter Host G, Kasbergen M, Truin GJ, Mulder J. Dental health in Dutch drug addicts. Community Dent Oral Epidemiol 1996;24:117-9.

25. Baratta C, Schrooten W, Colebunders R y cols. Utilization of healthcare services by people living with HIV/AIDS in Europe. Eurosupport Group. Int J STD AIDS 2000;11:784-9.

26. Ter Horst G, Molendijk B, Brower E, Verhey HG. Differences in dental treatment plan an planning for drug-addicted and non-drugaddicted patients. Community Dent Oral Epidemiol 1996;24:120-3.

27. Maskarenhas AK, Smith SR. Access use of specific dental services in HIV disease. J Public Health Dent 2000;60:172-81.

28. Gallagher PD, Gealer M, Birnbaum W. Resource implications for oral care of patients with HIV. Oral Dis 1998;4:22-5. 